\title{
On the continuity of spectra for families of magnetic pseudodifferential operators
}

\author{
Nassim Athmouni, ${ }^{1}$ Marius Măntoiu, ${ }^{2}$ and Radu Purice ${ }^{3}$ \\ 1) Faculté des Sciences de l'Université de Sfax, Sfax, Tunisie \\ 2) Departamento de Matemáticas, Universidad de Chile, Las Palmeras 3425, \\ Casilla 653, Santiago, Chile, Email: mantoiu@imar.ro \\ ${ }^{3)}$ Institute of Mathematics Simion Stoilow of the Romanian Academy, \\ P.O. Box 1-764, Bucharest, RO-70700, Romania, Email: purice@imar.ro
}

(Dated: 19 October 2018)

For families of magnetic pseudodifferential operators defined by symbols and magnetic fields depending continuously on a real parameter $\epsilon$, we show that the corresponding family of spectra also varies continuously with $\epsilon$.

2000 Mathematics Subject Classification: 47A10, 47L65, 81Q10.

PACS numbers: 47A10, 47L65, 81Q10

Keywords: Magnetic field, pseudodifferential operator, spectrum, crossed product, continuous field, $C^{*}$-algebra 


\section{INTRODUCTION AND MAIN RESULT}

It is known $2,6,11,19$, that "the spectrum of a Schrödinger operator with magnetic field $B$ is continuous in $B$ " under some assumptions on the regularity of the magnetic field. Following some ideas in ${ }^{3}$ and $^{4}$, we would like to put this result in a more general (abstract) perspective.

We shall work on the phase space $\Xi:=\mathcal{X} \times \mathcal{X}^{*} \equiv \mathbb{R}^{n} \times \mathbb{R}^{n}$ and use systematically notations of the form $X=(x, \xi), Y=(y, \eta), \ldots$ for its points. We shall consider classical Hamiltonians $h: \Xi \rightarrow \mathbb{R}$ (not having a simple specific form), defined on the phase space, smooth magnetic fields $B$ (closed 2-forms with bounded derivatives of any order) and quantum Hamiltonians $H^{A} \equiv \mathfrak{O p}^{A}(h)$ defined by a choice of a vector potential $A$ (with $\left.B=d A\right)^{13,15,16}$. Our aim is to study the continuity properties of the spectrum $\sigma\left(H^{A}\right)$ as a subset of $\mathbb{R}$ when both the symbol and the magnetic field $B$ depend on a parameter $\epsilon$ belonging to some interval $I$.

The main obstacles are the general form of the symbols $h^{\epsilon}$ and the fact that $H^{A^{\epsilon}}$ is defined using the vector potential $A^{\epsilon}$ which can be rather bad behaved even for bounded and smooth magnetic fields $B^{\epsilon}$. To overcome this, we work only with the magnetic symbol of the operators $H^{A^{\epsilon}}$ and we obtain affiliation ${ }^{1,10}$ of the classical Hamiltonians $h^{\epsilon}$ to a certain (not locally trivial) continuous field ${ }^{7}$ of twisted crossed-product $C^{*}$-algebras ${ }^{21}$, defined only in terms of the magnetic fields $\left\{B^{\epsilon}\right\}_{\epsilon \in I}{ }^{17}$. In this way, the problem is reduced to the study of the continuity properties in $\epsilon$ of the magnetic symbols $r^{\epsilon}$ defining resolvent families of the operators $H^{A^{\epsilon}}$. Then the results in ${ }^{23}$ directly imply the outer continuity of the spectrum (i.e. the 'stability of the spectral gaps') and the strong continuity in the regular representation (that we shall prove) implies the inner continuity of the spectrum (i.e. the 'stability of spectral islands').

To describe this result, we start recalling our version of covariant quantization in a magnetic field. Given a continuous magnetic field $B=d A$ defined by a vector potential $A$, we have the following quantization rule ${ }^{12,13,15}$ :

$$
\left[\mathfrak{O p}^{A}(f) u\right](x):=(2 \pi)^{-n} \int_{\mathcal{X}} d y \int_{\mathcal{X}^{\star}} d \eta e^{i(x-y) \cdot \eta} \lambda^{A}(x, y) f\left(\frac{x+y}{2}, \eta\right) u(y),
$$

with

$$
\lambda^{A}(x, y):=e^{-i \int_{[x, y]} A}=e^{-i(y-x) \cdot \int_{0}^{1} d s A(x+s(y-x))} .
$$

This is first defined for $f$ belonging to the Schwartz space $\mathcal{S}(\Xi)$ but extends to a topological 
isomorphism $^{15}$

$$
\mathfrak{O p} \mathfrak{p}^{A}: \mathcal{S}^{\prime}(\Xi) \rightarrow \mathbb{B}\left(\mathcal{S}(\mathcal{X}) ; \mathcal{S}^{\prime}(\mathcal{X})\right)
$$

(with the dual Fréchet topology on $\mathcal{S}^{\prime}(\Xi)$ and the strong topology on $\mathbb{B}\left(\mathcal{S}(\mathcal{X}) ; \mathcal{S}^{\prime}(\mathcal{X})\right.$ )). The main reason to use (1) is gauge-covariance: equivalent choices of vector potentials lead to unitarily equivalent operators.

Our quantization induces on the algebra of observables a composition law that only depends on the magnetic field $B^{12,15}$, requiring

$$
\mathfrak{O} \mathfrak{p}^{A}(f) \mathfrak{O} \mathfrak{p}^{A}(g)=: \mathfrak{O} \mathfrak{p}^{A}\left(f \sharp^{B} g\right)
$$

for any $f, g \in \mathcal{S}(\Xi)$. Explicitly we have

$$
\left(f \sharp^{B} g\right)(X)=\pi^{-2 n} \int_{\Xi} \int_{\Xi} d Y d Z e^{-2 i \sigma(X-Y, X-Z)} \Omega^{B}(x, y, z) f(Y) g(Z),
$$

where $\sigma$ is the canonical symplectic form on $\Xi$ and $\Omega^{B}:=\exp \left\{-i \Gamma^{B}\right\}$ with $\Gamma^{B}(x, y, z)$ defined as the flux of the magnetic field through the triangle $\langle x-y-z, x+y-z, x-y+z\rangle$ :

$$
\Gamma^{B}(x, y, z):=4 \sum_{j, k=1}^{n} y_{j} z_{k} \int_{0}^{1} d s \int_{0}^{1-s} d t B_{j k}(x-y-z+2 s y+2 t z) .
$$

Extending $\sharp^{B}$ by duality, we get the magnetic Moyal algebra

$$
\mathfrak{M}^{B}(\Xi):=\left\{f \in \mathcal{S}^{\prime}(\Xi) \mid \forall g \in \mathcal{S}(\Xi), f \sharp^{B} g \in \mathcal{S}(\Xi), g \sharp^{B} f \in \mathcal{S}(\Xi)\right\}
$$

and the algebra of bounded observables

$$
\mathfrak{A}^{B}(\Xi):=\left(\mathfrak{O p}^{A}\right)^{-1}\left[\mathbb{B}\left(L^{2}(\mathcal{X})\right)\right]
$$

This second one will be a $C^{*}$-algebra isomorphic to $\mathbb{B}\left(L^{2}(\mathcal{X})\right)$; it depends on the magnetic field but not on the vector potential, by gauge covariance.

Our aim is to show how our intrinsic observable algebra approach to the study of quantum Hamiltonians in non-homogenuous magnetic fields allows for a proof of the continuity of the spectra with respect to very general variations of the symbol and of the magnetic field.

Let us state the assumptions. We need Hörmander's classes of symbols

$$
S_{\rho}^{m}(\Xi):=\left\{f \in C^{\infty}(\Xi)\left|\forall(a, \alpha) \in \mathbb{N}^{n} \times \mathbb{N}^{n}, \exists C_{a \alpha}>0,\right|\left(\partial_{x}^{a} \partial_{\xi}^{\alpha} f\right)(x, \xi) \mid \leq C_{a \alpha}\langle\xi\rangle^{m-\rho|\alpha|}\right\},
$$

where $\langle\xi\rangle:=\left(1+|\xi|^{2}\right)^{1 / 2}$. Our previous results ${ }^{12,15}$ show that $S_{\rho}^{m}(\Xi) \subset \mathfrak{M}^{B}(\Xi)$ and $S_{0}^{0}(\Xi) \subset$ $\mathfrak{A}^{B}(\Xi)$.

On the symbol spaces $(m \in \mathbb{R}, \rho=1,0)$ we have Fréchet structures defined by the families of semi-norms indexed by $N, M \in \mathbb{N}$ 
- $\|f\|_{(\Xi, 1, m, N, M)}:=\max _{|a| \leq N|\alpha| \leq M} \operatorname{map}_{(x, \xi)}\left|\langle\xi\rangle^{-m+|\alpha|}\left(\partial_{x}^{a} \partial_{\xi}^{\alpha} f\right)(x, \xi)\right|, \quad \forall f \in S_{1}^{m}(\Xi)$,

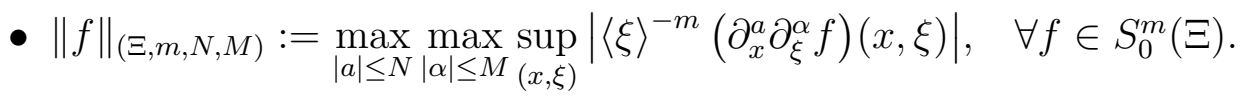

We also recall that $f \in S_{1}^{m}(\Xi)$ is called elliptic (and we write $f \in S_{1, \text { ell }}^{m}(\Xi)$ ) if

$$
|f(x, \xi)| \geq C\langle\xi\rangle^{m} \quad \text { for }|\xi| \text { big enough. }
$$

Hypothesis 1.1. Consider a family of Hamiltonians $\left\{h^{\epsilon}\right\}_{\epsilon \in I}$ with $I \subset \mathbb{R}$ a compact interval, such that

- $h^{\epsilon} \in S_{1, \text { ell }}^{m}(\Xi)$ with $m>0$, for each $\epsilon \in I$,

- the map $I \ni \epsilon \mapsto h^{\epsilon} \in S_{1}^{m}(\Xi)$ is continuous for the Fréchet topology on $S_{1}^{m}(\Xi)$.

- there exist $C \in \mathbb{R}$ such that $h^{\epsilon} \geq-C, \forall \epsilon \in I$.

Hypothesis 1.2. We are given a family of magnetic fields $\left\{B^{\epsilon}\right\}_{\epsilon \in I}$ with the components $B_{j k}^{\epsilon} \in B C^{\infty}(\mathcal{X})$ such that the map $I \ni \epsilon \mapsto B_{j k}^{\epsilon} \in B C^{\infty}(\mathcal{X})$ is continuous for the Fréchet topology on $B C^{\infty}(\mathcal{X})$.

It has been shown in $^{12}$ that real elliptic elements $f$ of $S_{1}^{m}(\Xi)$ define self-adjoint operators $\mathfrak{O p}^{A}(f)$ in the Hilbert space $\mathcal{H}:=L^{2}(\mathcal{X})$, having as domain a suitable magnetic analog of the $m^{\prime}$ th order Sobolev space. The semi-norms on $B C^{\infty}(\mathcal{X})$ can be obtained from the expressions above for $\|\cdot\|_{(\Xi, m, N, M)}$, by replacing $\Xi$ with $\mathcal{X}$ and by setting $m=0$.

In order to state our main result we recall some notions of continuity of subsets ${ }^{3,4}$.

Definition 1.3. Let $I$ be a compact interval and suppose given a family $\left\{\sigma^{\epsilon}\right\}_{\epsilon \in I}$ of closed subsets of $\mathbb{R}$.

1. The family $\left\{\sigma^{\epsilon}\right\}_{\epsilon \in I}$ is called outer continuous at $\epsilon_{0} \in I$ if for any compact subset $K$ of $\mathbb{R}$ such that $K \cap \sigma^{\epsilon_{0}}=\emptyset$, there exists a neighborhood $V_{K}^{\epsilon_{0}}$ of $\epsilon_{0}$ with $K \cap \sigma^{\epsilon}=\emptyset$, $\forall \epsilon \in V_{K}^{\epsilon_{0}}$.

2. The family $\left\{\sigma^{\epsilon}\right\}_{\epsilon \in I}$ is called inner continuous at $\epsilon_{0} \in I$ if for any open subset $\mathcal{O}$ of $\mathbb{R}$ such that $\mathcal{O} \cap \sigma^{\epsilon_{0}} \neq \emptyset$, there exists a neighborhood $V_{\mathcal{O}}^{\epsilon_{0}} \subset I$ of $\epsilon_{0}$ with $\mathcal{O} \cap \sigma^{\epsilon} \neq \emptyset$, $\forall \epsilon \in V_{\mathcal{O}}^{\epsilon_{0}}$. 
$\operatorname{In}^{3}$ the sets $\sigma^{\epsilon}$ are compact and $K$ is only taken to be closed.

Theorem 1.4. Suppose given a compact interval $I \subset \mathbb{R}$, a family of classical Hamiltonians $\left\{h^{\epsilon}\right\}_{\epsilon \in I}$ satisfying Hypothesis 1.1 and a family of magnetic fields $\left\{B^{\epsilon}\right\}_{\epsilon \in I}$ satisfying Hypothesis 1.2. Let us consider the family of quantum Hamiltonians $H^{\epsilon}:=\mathfrak{O p}^{A^{\epsilon}}\left(h^{\epsilon}\right)$ for some choice of a vector potential $A^{\epsilon}$ for $B^{\epsilon}$. Then the spectra $\sigma^{\epsilon}:=\sigma\left(H^{\epsilon}\right) \subset \mathbb{R}$ form an outer and inner continuous family at any point $\epsilon \in I$.

Of course, if one only asks continuity conditions on the families $\left\{B^{\epsilon}\right\}_{\epsilon \in I}$ and $\left\{h^{\epsilon}\right\}_{\epsilon \in I}$ at some point $\epsilon_{0} \in I$, the (outer and inner) continuity of the family of spectra will only be guaranteed at $\epsilon_{0}$.

Let us briefly comment upon the significance of Theorem 1.4:

- It extends the results in $^{3,8}$ to the case of continuous models (with configuration space $\mathcal{X}=\mathbb{R}^{n}$ ) and non-constant magnetic fields. We mention in this context that our objects are no longer elements of a crossed product but only unbounded observables affiliated to twisted crossed-products with twisting cocycle in a rather complicated (not locally compact) group.

- It extends the known results ${ }^{11,19}$ to the class of elliptic symbols of any form and of any strictly positive order. Notice that for Schrödinger type operators $\left(h^{\epsilon}(x, \xi)=\right.$ $\left.\xi^{2}+V^{\epsilon}(x)\right)$ the condition that the components of the magnetic field should be smooth may be very much weakened as we are going to show in future publication.

- It is the first step in the study of the regularity of the spectral bands and gaps with respect to variation of the magnetic field $\left(\mathrm{see}^{3}\right)$.

Our paper is devoted to the proof of Theorem 1.4 and has the following structure. In the next Section we present an abstract argument (following ideas and arguments in ${ }^{3,8}$ ) relating the statement of Theorem 1.4 to the continuity of the symbols of the resolvents of the family $\left\{H^{\epsilon}\right\}_{\epsilon \in I}$ in some special family of $C^{*}$-algebras, reducing the proof of Theorem 1.4 to that of Theorem 2.7. The third Section is devoted to our main technical result proving the affiliation of the family $\left\{H^{\epsilon}\right\}_{\epsilon \in I}$ to a specific twisted crossed product $C^{*}$-algebra. In the 4-th Section we use the results in $^{23}$ to prove that this last twisted crossed product $C^{*}$-algebra is in fact an algebra of continuous sections in a field of $C^{*}$-algebras and this is shown to be equivalent to our Theorem 2.7, thus finishing the proof of Theorem 1.4. 


\section{THE ABSTRACT PART OF THE PROOF}

The abstract step in proving Theorem 1.4 is to show how the norm of the resolvent $R^{\epsilon}(\mathfrak{z}):=\left(H^{\epsilon}-\mathfrak{z} 1\right)^{-1}$ is relevant for spectral continuity. In fact we have the following result. Proposition 2.5. Suppose that $\left\{H^{\epsilon}\right\}_{\epsilon \in I}$ is a family of self-adjoint operators in the Hilbert space $\mathcal{H}$ such that for any $\mathfrak{z} \notin \mathbb{R}$ the map

$$
I \ni \epsilon \mapsto\left\|\left(H^{\epsilon}-\mathfrak{z} 1\right)^{-1}\right\| \in \mathbb{R}_{+}
$$

is upper (resp. lower) semi-continuous in $\epsilon_{0} \in I$. Then the spectra $\left\{\sigma\left(H^{\epsilon}\right)\right\}_{\epsilon \in I}$ form an outer (resp. inner) continuous family of closed sets at the point $\epsilon_{0} \in I$.

Corollary 2.6. If for any $\mathfrak{z} \notin \mathbb{R}$ the map

$$
I \ni \epsilon \mapsto\left\|\left(H^{\epsilon}-\mathfrak{z} 1\right)^{-1}\right\| \in \mathbb{R}_{+}
$$

is continuous, then the family $\left\{\sigma\left(H^{\epsilon}\right)\right\}_{\epsilon \in I}$ is both outer and inner continuous.

Proof. For any $\mathfrak{z} \in \mathbb{C} \backslash \mathbb{R}$ the functions $\kappa_{\mathfrak{z}}(t):=(t-\mathfrak{z})^{-1}$ belong to $C_{0}(\mathbb{R})$, i.e. they are continuous and small at infinity. Due to the Stone-Weierstrass Theorem and the resolvent equation, their linear span is in fact an algebra and is dense in $C_{0}(\mathbb{R})$ for the norm $\|\cdot\|_{\infty}$. Thus, for any $\chi \in C_{0}(\mathbb{R})$ and for any $\delta>0$, there exist $N \in \mathbb{N}, a_{j} \in \mathbb{C}$ and $\mathfrak{z}_{j} \in \mathbb{C} \backslash \mathbb{R}$, with $j \in\{1, \ldots, N\}$, such that

$$
\left\|\chi-\sum_{j=1}^{N} a_{j} \kappa_{\mathfrak{z} j}\right\|_{\infty} \leq \delta .
$$

By the functional calculus for self-adjoint operators we have $R^{\epsilon}(\mathfrak{z})=\kappa_{\mathfrak{z}}\left(H^{\epsilon}\right)$. We infer that for any $\chi \in C_{0}(\mathbb{R})$ the map $I \ni \epsilon \mapsto\left\|\chi\left(H^{\epsilon}\right)\right\| \in \mathbb{R}_{+}$has the same semi-continuity property as the map $I \ni \epsilon \mapsto\left\|R^{\epsilon}(\mathfrak{z})\right\| \in \mathbb{R}_{+}$.

Let us suppose now upper semi-continuity in $\epsilon_{0}$ and assume that $\sigma\left(H^{\epsilon_{0}}\right) \cap K=\emptyset$ for some compact set $K$. By Urysohn's Lemma, there exists $\chi \in C_{0}(\mathbb{R})_{+}$with $\left.\chi\right|_{K}=1$ and $\left.\chi\right|_{\sigma\left(H_{0}\right)}=0$, so $\chi\left(H^{\epsilon_{0}}\right)=0$. Choose a neighborhood $V$ of $\epsilon_{0}$ such that for $\epsilon \in V$

$$
\left\|\chi\left(H^{\epsilon}\right)\right\| \leq\left\|\chi\left(H^{\epsilon_{0}}\right)\right\|+\frac{1}{2}=\frac{1}{2} .
$$

If for some $\epsilon \in V$ there exists $\lambda \in K \cap \sigma\left(H^{\epsilon}\right)$, then

$$
1=\chi(\lambda) \leq \sup _{\mu \in \sigma^{\epsilon}} \chi(\mu)=\left\|\chi\left(H^{\epsilon}\right)\right\| \leq \frac{1}{2},
$$


which is absurd.

Let us assume now lower semi-continuity in $\epsilon_{0}$. Suppose that there exist an open set $\mathcal{O} \subset \mathbb{R}$ such that $\sigma\left(H^{\epsilon_{0}}\right) \cap \mathcal{O} \neq \emptyset$ and let $\lambda \in \sigma^{\epsilon} \cap \mathcal{O}$. By Urysohn's Lemma there exist a positive function $\chi \in C_{0}(\mathbb{R})$ with $\chi(\lambda)=1$ and $\operatorname{supp}(\chi) \subset \mathcal{O}$; thus $\left\|\chi\left(H^{\epsilon_{0}}\right)\right\| \geq 1$. Suppose moreover that for any neighborhood $V \subset I$ of $\epsilon_{0}$ there exists $\epsilon \in V$ such that $\sigma\left(H^{\epsilon}\right) \cap \mathcal{O}=\emptyset$ and thus $\chi\left(H^{\epsilon}\right)=0$. This clearly contradicts the lower semi-continuity of $\epsilon \mapsto\left\|\chi\left(H^{\epsilon}\right)\right\|$. We conclude thus the inner continuity condition.

Proving the continuity of the map $I \ni \epsilon \mapsto\left\|R^{\epsilon}(\mathfrak{z})\right\| \in \mathbb{R}_{+}$for any $\mathfrak{z} \in \mathbb{C} \backslash \mathbb{R}$ is the aim of the remaining part of the article. Our approach will be to work intrinsically with the symbol $r_{\mathfrak{z}}^{\epsilon}=\left(\mathfrak{O p}^{A^{\epsilon}}\right)^{-1}\left[R^{\epsilon}(\mathfrak{z})\right]$. Clearly $r_{\mathfrak{z}}^{\epsilon} \in \mathfrak{A}^{\epsilon}(\Xi) \equiv \mathfrak{A}^{B^{\epsilon}}(\Xi)$ (it depends on the parameter $\epsilon \in I$ both through the $\epsilon$-dependence of the symbol $h^{\epsilon}$ and through the $\epsilon$-dependence of the product $\sharp^{\epsilon}$, which is $B^{\epsilon}$-dependent) and $\left\|r_{\mathfrak{z}}^{\epsilon}\right\|_{\epsilon}=\left\|R^{\epsilon}(\mathfrak{z})\right\|$ will be now an $\epsilon$-dependent norm. However, no vector potential is in view now. Our main technical result, Proposition 3.10, proven in the next section allows us to control the inverse $r_{\mathfrak{z}}^{\epsilon}$ of $h^{\epsilon}-\mathfrak{z} 1$ in the Moyal algebra $\mathfrak{M}^{\epsilon}(\Xi):=\mathfrak{M}^{B^{\epsilon}}(\Xi)$ with respect to the product $\sharp \epsilon \equiv \sharp^{B^{\epsilon}}$ for $\epsilon$ fixed (a problem of affiliation), to prove that it belongs in fact to some smaller algebra and to control the $\epsilon$-dependence of the norms of the elements $r_{\mathfrak{z}}^{\epsilon} \in \mathfrak{C}^{\epsilon} \subset \mathfrak{A}^{\epsilon}(\Xi)$. These results will allow us to place ourselves in the setting of continuous fields of $C^{*}$-algebras. We shall prove the following statement

Theorem 2.7. Suppose given a family of symbols $\left\{h^{\epsilon}\right\}_{\epsilon \in I}$ satisfying Hypothesis 1.1 and a family of magnetic fields $\left\{B^{\epsilon}\right\}_{\epsilon \in I}$ satisfying Hypothesis 1.2, then, for any choice of vector potentials $\left\{A^{\epsilon}\right\}_{\epsilon \in I}$ associated to the magnetic fields $B^{\epsilon}\left(B^{\epsilon}=d A^{\epsilon}\right)$ and for any $\mathfrak{z} \in \mathbb{C} \backslash \mathbb{R}$ the map

$$
I \ni \epsilon \mapsto\left\|\left(\mathfrak{O p}^{A^{\epsilon}}\left(h^{\epsilon}\right)-\mathfrak{z} 1\right)^{-1}\right\| \in \mathbb{R}_{+}
$$

is continuous.

Thus, by the discussion above, we conclude that our Theorem 1.4 is true.

The main tool in proving Theorem 2.7 will be to embed our symbol algebras depending on $\epsilon \in I$ as "continuous sections in the direct product" $\coprod_{\epsilon \in I} \mathfrak{A}^{\epsilon}(\Xi) \rightarrow I$. We shall constantly use the notation $B C_{u}(\mathcal{X})$ for the abelian $C^{*}$-algebra of all bounded uniformly continuous complex functions on $\mathcal{X}$. We shall construct these "continuous sections" by considering the twisted crossed-products $B C_{u}(\mathcal{X}) \rtimes_{\theta}^{\omega^{\epsilon}} \mathcal{X}$ for each $\epsilon \in I$ (studied in ${ }^{15,17}$ ); here $\theta$ denotes the natural action of $\mathcal{X}$ on $B C_{u}(\mathcal{X})$ by translations and $\omega^{\epsilon} \equiv \omega^{B^{\epsilon}}$ is a group 2-cocycle to be 
introduced below.

Let us consider the inverse partial Fourier transform

$$
\mathfrak{F}^{-}: \mathcal{S}(\Xi) \rightarrow \mathcal{S}(\mathcal{X} \times \mathcal{X}), \quad\left[\mathfrak{F}^{-} f\right](x, y):=\int_{\mathcal{X}^{*}} d \xi e^{i \xi \cdot y} f(x, \xi)
$$

(extended to $\mathcal{S}^{\prime}(\Xi)$ and $L^{2}(\Xi)$ ). We can transport the Moyal product $\sharp^{B}$ to a bilinear associative product on $\mathcal{S}(\mathcal{X} \times \mathcal{X})$ and $\mathfrak{F}^{-} \mathfrak{M}^{B}(\Xi)$ that we denote by $\diamond^{B}$ :

$$
\phi \diamond^{B} \psi:=\mathfrak{F}^{-}\left[(\mathfrak{F} \phi) \sharp^{B}(\mathfrak{F} \psi)\right] .
$$

A simple computation gives

$$
\left[\phi \diamond^{B} \psi\right](x, y)=\int_{\mathcal{X}} d z \phi(x+(z-y) / 2, z) \psi(x+z / 2, y-z) \omega^{B}(x-y / 2 ; z, y-z)
$$

where

$$
\omega^{B}(x ; y, z):=\exp \left\{\left(-i \gamma^{B}(x, y, z)\right)\right\} \in C(\mathcal{X} ; \mathbb{U}(1))
$$

and $\gamma^{B}(x, y, z)$ is the flux of $B$ through the triangle $<x, x+y, x+y+z>$. The group $C(\mathcal{X} ; U(1))$ can be identified with the group of all the unitary elements in the $C^{*}$-algebra $B C_{u}(\mathcal{X})$.

It is easy to see that the Banach space $\mathfrak{L}:=L^{1}\left(\mathcal{X} ; B C_{u}(\mathcal{X})\right)$ is contained in $\mathfrak{F}^{-}\left[\mathfrak{A}^{B}(\Xi)\right]$ and is also a Banach ${ }^{*}$-algebra under the multiplication $\diamond^{B}$ and the involution given by

$$
\phi^{*}(x ; y) \equiv\left[\phi^{*}(y)\right](x):=\overline{\phi(x ;-y)}
$$

Let $\mathfrak{C}^{B}$ be the closure of $\mathfrak{F}(\mathfrak{L})$ in $\mathfrak{A}^{B}(\Xi)$ (with the product $\sharp^{B}$ ). We shall also consider the $C^{*}$-algebra $\mathfrak{B}^{B}:=\mathfrak{F}^{-} \mathfrak{C}^{B}$ (for the product $\diamond^{B}$ ), that will be contained in $\mathcal{S}^{\prime}(\mathcal{X} \times \mathcal{X})$. This $C^{*}$-algebra is exactly the twisted crossed-product $B C_{u}(\mathcal{X}) \rtimes_{\theta}^{\omega^{B}} \mathcal{X}$ associated to $B C_{u}(\mathcal{X})$, the action $\theta$ by translations of $\mathcal{X}$ on $B C_{u}(\mathcal{X})$ and the 2-cocycle $\omega^{B 17,21,22}$ and also the enveloping $C^{*}$-algebra of the Banach *-algebra $\mathfrak{L}$. Let us strengthen that the two $C^{*}$-algebras $\mathfrak{B}^{B}$ and $\mathfrak{C}^{B}$ are isomorphic. The constructions above can be performed for any of the magnetic fields $B^{\epsilon}, \epsilon \in I$. We are going to use the abbreviations $\mathfrak{C}^{\epsilon}:=\mathfrak{C}^{B^{\epsilon}}, \mathfrak{B}^{\epsilon}:=\mathfrak{B}^{B^{\epsilon}}$.

In estimating some $C^{*}$-norms we shall need a special representation, the left regular representation

$$
\Pi^{\epsilon}: \mathfrak{B}^{\epsilon} \rightarrow \mathbb{B}\left[L^{2}(\mathcal{X} \times \mathcal{X})\right], \quad \Pi^{\epsilon}(\phi) \psi:=\phi \diamond^{\epsilon} \psi, \quad \forall \phi \in \mathfrak{B}^{\epsilon}, \quad \forall \psi \in \mathcal{H}:=L^{2}(\mathcal{X} \times \mathcal{X})
$$

It really defines a representation, as one can easily notice using the results in ${ }^{15}$. 
We close this section by introducing a new algebra of $\epsilon$-dependent symbols. We want to 'glue' all the 2-cocycles $\omega^{\epsilon}(y, z) \in C(\mathcal{X} ; U(1))$ for $\epsilon \in I$ in a single 2-cocycle with values in a larger group $C(I \times \mathcal{X} ; U(1))$. This obliges us to also enlarge the unital abelian algebra $B C_{u}(\mathcal{X})$ to the unital abelian algebra $B C_{u}(I \times \mathcal{X})=C\left(I ; B C_{u}(\mathcal{X})\right)$. Let us consider the

natural action $\widetilde{\theta}$ by translations of $\mathcal{X}$ on $C\left(I ; B C_{u}(\mathcal{X})\right)$, given explicitely by $[\widetilde{\theta}(x) f](\epsilon, z):=$ $f(\epsilon, z+x)$, the 2-cocycle $\widetilde{\omega}: \mathcal{X} \times \mathcal{X} \rightarrow C(I \times \mathcal{X} ; U(1))$ given by

$$
[\widetilde{\omega}(y, z)](\epsilon, x):=\left[\omega^{\epsilon}(y, z)\right](x)
$$

and the following composition law (similar to $(10))$ on $C_{c}\left(\mathcal{X} ; C\left(I ; B C_{u}(\mathcal{X})\right)\right)$ :

$$
[\widetilde{\phi} \diamond \widetilde{\psi}](\epsilon, x, y):=\int_{\mathcal{X}} d z \widetilde{\phi}(\epsilon, x+(z-y) / 2, z) \widetilde{\psi}(\epsilon, x+z / 2, y-z)[\widetilde{\omega}(z, y-z)](\epsilon, x-y / 2) .
$$

Taking the closure of $C_{c}\left(\mathcal{X} ; C\left(I ; B C_{u}(\mathcal{X})\right)\right)$ with respect to the norm

$\|\widetilde{\phi}\|_{\tilde{\mathcal{L}}}:=\int_{\mathcal{X}} d x\|\widetilde{\phi}(x)\|_{C\left(I ; B C_{u}(\mathcal{X})\right)}=\int_{\mathcal{X}} d x \sup _{\epsilon \in I}\|[\widetilde{\phi}(x)](\epsilon)\|_{B C_{u}(\mathcal{X})}=\int_{\mathcal{X}} d x \sup _{\epsilon \in I} \sup |[\widetilde{\phi}(x)](\epsilon ; y)|$

we obtain the space $\widetilde{\mathcal{L}}=L^{1}\left(\mathcal{X} ; C\left(I ; B C_{u}(\mathcal{X})\right)\right)$ that is a Banach algebra for the composition (14). Let us consider its $C^{*}$-envelope that will be a crossed-product

$$
\mathfrak{B}:=C\left(I ; B C_{u}(\mathcal{X})\right) \rtimes_{\widetilde{\omega}}^{\widetilde{X}}
$$

We can then also define the isomorphic $C^{*}$-algebra $\mathfrak{C}:=\mathfrak{F}[\mathfrak{B}]$.

An important remark is that for any $\epsilon \in I$ we have a natural evaluation map

$$
\mathfrak{e}_{\epsilon}: C_{c}\left(\mathcal{X} ; C\left(I ; B C_{u}(\mathcal{X})\right)\right) \rightarrow C_{c}\left(\mathcal{X} ; B C_{u}(\mathcal{X})\right), \quad \mathfrak{e}_{\epsilon}(\widetilde{\phi})(x):=[\widetilde{\phi}(x)](\epsilon) \in B C_{u}(\mathcal{X}),
$$

that extends by continuity to a contractive and surjective projection (that we shall denote by the same symbol) $\mathfrak{e}_{\epsilon}: \widetilde{\mathcal{L}} \rightarrow \mathcal{L}$ and to a contractive $C^{*}$-homomorphism $\mathfrak{e}_{\epsilon}: \mathfrak{B} \rightarrow \mathfrak{B}^{\epsilon}$.

\section{AN AFFILIATION RESULT}

As mentioned in the Introduction, in this Section we prove the affiliation of our family of Hamiltonians $\left\{H^{\epsilon}\right\}_{\epsilon \in I}$ to a specific twisted crossed product $C^{*}$-algebra. Unfortunately, no one of the affiliation results we have proved already $\left(\mathrm{see}^{18},{ }^{14}\right)$ implies directly Proposition 3.10. Therefore we decided to give a full proof of the statement. We shall mainly follow the 
arguments in $^{18}$, keeping trace of the $\epsilon$-dependence and adding the necessary technicalities in order to deal with $x$-dependent symbols.

We first need notations for the norms defining the Fréchet topologies on various spaces. Let $C_{\text {pol }}^{\infty}(\mathcal{X})$ be the space of indefinitely differentiable functions having polynomial growth as well as their derivatives. For $\varphi \in C_{\text {pol }}^{\infty}(\mathcal{X})$, with $p \in \mathbb{R}$ and $N \in \mathbb{N}$, we denote

$$
\|\varphi\|_{(\mathcal{X}, p, N)}:=\max _{|a| \leq N} \sup _{x \in \mathcal{X}}\left|\langle x\rangle^{-p}\left(\partial_{x}^{a} \varphi\right)(x)\right|
$$

thus the family $\|\varphi\|_{(\mathcal{X}, N)}:=\|\varphi\|_{(\mathcal{X}, 0, N)}$, with $N \in \mathbb{N}$, defines the Fréchet topology on $B C^{\infty}(\mathcal{X})$; we also denote by $\|\varphi\|_{\infty}:=\|\varphi\|_{(\mathcal{X}, 0)}$ the usual norm on $B C(\mathcal{X})$. Associated to the above norms we can also consider

$$
\|\phi\|_{(\mathcal{X}, p, N),(\mathcal{X}, q, M)}:=\max _{|a| \leq N|b| \leq M} \max _{y \in \mathcal{X}} \sup _{z \in \mathcal{X}}\left|\langle y\rangle^{-p}\langle z\rangle^{-q}\left(\partial_{y}^{a} \partial_{z}^{b} \phi\right)(y, z)\right|
$$

for all $\phi \in C_{\text {pol }}^{\infty}(\mathcal{X} \times \mathcal{X})$, for $p, q \in \mathbb{R}$ and $N, M \in \mathbb{N}$ and

$$
\|\widetilde{\phi}\|_{\infty,(\mathcal{X}, p, N),(\mathcal{X}, q, M)}:=\sup _{x \in \mathcal{X}}\|\widetilde{\phi}(x)\|_{(\mathcal{X}, p, N),(\mathcal{X}, q, M)}
$$

for any $\widetilde{\phi} \in B C\left(\mathcal{X} ; C_{\text {pol }}^{\infty}(\mathcal{X} \times \mathcal{X})\right)$. For $F \in S_{1,1}^{k_{1}, k_{2}}(\Xi \times \Xi)$ we shall need the following family of norms

$$
\begin{gathered}
\|F\|_{\left(k_{1}, N_{1}, M_{1}\right),\left(k_{2}, N_{2}, M_{2}\right)}:= \\
\max _{|a| \leq N_{1}} \max _{|\alpha| \leq M_{1}} \max _{|b| \leq N_{2}} \max _{|\beta| \leq M_{2}} \sup _{Y \in \Xi} \sup _{Z \in \Xi}\left|<\eta>^{-k_{1}+|\alpha|}<\zeta>^{-k_{2}+|\beta|}\left(\partial_{y}^{a} \partial_{\eta}^{\alpha} \partial_{z}^{b} \partial_{\zeta}^{\beta} F^{\epsilon}\right)(Y, Z)\right| .
\end{gathered}
$$

Now let us introduce the main technical tool that will allow us to estimate the oscillating integrals appearing in the computation of the symbol of the resolvent.

Lemma 3.8. We consider a function $\gamma \in C\left(I ; B C^{\infty}\left(\mathcal{X} ; C_{\mathrm{pol}}^{\infty}(\mathcal{X} \times \mathcal{X})\right)\right)$ satisfying estimations of the form

$$
C(\gamma) \equiv \sup _{\epsilon \in I}\left\|\partial_{x}^{\alpha} \gamma^{\epsilon}\right\|_{\infty,\left(\mathcal{X}, s_{1}\left(N_{1}, N_{2}\right), N_{1}\right),\left(\mathcal{X}, s_{2}\left(N_{1}, N_{2}\right), N_{2}\right)}<\infty, \quad \forall \alpha \in \mathbb{N}^{n}
$$

and a function $F_{\lambda} \in C\left(I ; B C^{\infty}\left(\Xi ; S_{1,1}^{k_{1} k_{2}}(\Xi \times \Xi)\right)\right)$ satisfying estimations of the form

$$
\sup _{\epsilon \in I} \sup _{x \in \mathcal{X}}\left\|\left[\partial_{x}^{b} \partial_{\xi}^{\alpha} F_{\lambda}^{\epsilon}\right](X)\right\|_{\left(k_{1}, N_{1}, M_{1}\right),\left(k_{2}, N_{2}, M_{2}\right)} \leq C_{a, \beta}\left(F_{\lambda}\right)\langle\xi\rangle^{-\rho-|\beta|} \lambda^{-\rho^{\prime}}
$$

for some strictly positive exponents $\rho$ and $\rho^{\prime}$. Then the function

$$
G(\epsilon ; \lambda ; X):=\int_{\Xi} \int_{\Xi} d Y d Z e^{-2 i z \cdot \eta} e^{2 i y \cdot \zeta} \gamma^{\epsilon}(x ; y, z) F_{\lambda}^{\epsilon}(X ; Y, Z)
$$


defines for each $\lambda>0$ an element of $C\left(I ; S_{1}^{-\rho}(\Xi)\right)$ and we have

$$
\sup _{\epsilon \in I}\|G(\epsilon ; \lambda ; \cdot)\|_{\Xi, 1,-\rho, N, M} \leq C \lambda^{-\rho^{\prime}} .
$$

Proof. We shall introduce some integrable factors into the integral (17) by applying suitable differential operators to the phase factor $e^{-2 i z \cdot \eta} e^{2 i y \cdot \zeta}$. In fact we have

$$
\begin{aligned}
& \langle y\rangle^{-2}\left(1+\frac{1}{2 i} y \cdot \partial_{\zeta}\right) e^{2 i y \cdot \zeta}=e^{2 i y \cdot \zeta}, \quad\langle z\rangle^{-2}\left(1-\frac{1}{2 i} z \cdot \partial_{\eta}\right) e^{-2 i z \cdot \eta}=e^{-2 i z \cdot \eta}, \\
& \langle\eta\rangle^{-2}\left(1-\frac{1}{2 i} \eta \cdot \partial_{z}\right) e^{-2 i z \cdot \eta}=e^{-2 i z \cdot \eta}, \quad\langle\zeta\rangle^{-2}\left(1+\frac{1}{2 i} \zeta \cdot \partial_{y}\right) e^{2 i y \cdot \zeta}=e^{2 i y \cdot \zeta} .
\end{aligned}
$$

We integrate by parts in (17), first with respect to the $(y, z)$ variables (obtaining the integrable powers in $\eta$ and $\zeta$ and some growing factors in $(y, z))$ and then with respect to the $(\eta, \zeta)$ variables obtaining the integrable factors in $y$ and $z$ due to the symbol behavior of the function $F_{\lambda}$. More precisely, after $N_{1}+N_{2}+M_{1}+M_{2}$ integration by parts we obtain the equality:

$$
\begin{gathered}
\int_{\Xi} \int_{\Xi} d Y d Z e^{-2 i z \cdot \eta} e^{2 i y \cdot \zeta} \gamma^{\epsilon}(x ; y, z) F_{\lambda}^{\epsilon}(X ; Y, Z)= \\
=\int_{\Xi} \int_{\Xi} d Y d Z e^{-2 i z \cdot \eta} e^{2 i y \cdot \zeta} \times\left[\langle y\rangle^{-2 M_{2}}\left(1-\frac{1}{2 i} y \cdot \partial_{\zeta}\right)^{M_{2}}\langle z\rangle^{-2 M_{1}}\left(1+\frac{1}{2 i} z \cdot \partial_{\eta}\right)^{M_{1}} \times\right. \\
\left.\times\langle\eta\rangle^{-2 N_{2}}\left(1+\frac{1}{2 i} \eta \cdot \partial_{z}\right)^{N_{2}}\langle\zeta\rangle^{-2 N_{1}}\left(1-\frac{1}{2 i} \zeta \cdot \partial_{y}\right)^{N_{1}}\left(\gamma^{\epsilon} F_{\lambda}^{\epsilon}\right)\right](X ; Y, Z)= \\
=\int_{\Xi} \int_{\Xi} d Y d Z e^{-2 i z \cdot \eta} e^{2 i y \cdot \zeta} \times\left[\langle y\rangle^{-M_{2}}\langle z\rangle^{-M_{1}}\left(\frac{1}{\langle y\rangle}-\frac{y}{2 i\langle y\rangle} \cdot \partial_{\zeta}\right)^{M_{2}}\left(\frac{1}{\langle z\rangle}+\frac{z}{2 i\langle z\rangle} \cdot \partial_{\eta}\right)^{M_{1}} \times\right. \\
\left.\times\langle\eta\rangle^{-N_{2}}\langle\zeta\rangle^{-N_{1}}\left(\frac{1}{\langle\eta\rangle}+\frac{\eta}{2 i\langle\eta\rangle} \cdot \partial_{z}\right)^{N_{2}}\left(\frac{1}{\langle\zeta\rangle}-\frac{\zeta}{2 i\langle\zeta\rangle} \cdot \partial_{y}\right)^{N_{1}}\left(\gamma^{\epsilon} F_{\lambda}^{\epsilon}\right)\right](X ; Y, Z),
\end{gathered}
$$

where the differential polynomials have coefficients of class $B C^{\infty}(\Xi)$. This clearly implies the estimation

$$
\begin{gathered}
\left|\int_{\Xi} \int_{\Xi} d Y d Z e^{-2 i z \cdot \eta} e^{2 i y \cdot \zeta} \gamma^{\epsilon}(x ; y, z) F_{\lambda}^{\epsilon}(X ; Y, Z)\right| \leq \\
\leq C_{1}\left(N_{1}, N_{2}, M_{1}, M_{2}\right) \sup _{\epsilon \in I}\left\|\gamma^{\epsilon}\right\|\left(\mathcal{X}, s_{1}\left(N_{1}, N_{2}\right), N_{1}\right),\left(\mathcal{X}, s_{2}\left(N_{1}, N_{2}\right), N_{2}\right) \\
\sup _{\epsilon \in I}\left\|F^{\epsilon}(X)\right\|_{\left(k_{1}, N_{1}, M_{1}\right),\left(k_{2}, N_{2}, M_{2}\right)} \times \\
\times \int_{\Xi} d Y \int_{\Xi} d Z\langle y\rangle^{-M_{2}}\langle z\rangle^{-M_{1}}\langle\eta\rangle^{-N_{2}}\langle\zeta\rangle^{-N_{1}}\langle y\rangle^{s_{1}\left(N_{1}, N_{2}\right)}\langle z\rangle^{s_{2}\left(N_{1}, N_{2}\right)}\langle\eta\rangle^{k_{1}}\langle\zeta\rangle^{k_{2}} \leq \\
\leq C\left(N_{1}, N_{2}, M_{1}, M_{2}\right) C(\gamma) C\left(F_{\lambda}\right)\langle\xi\rangle^{-\rho} \lambda^{-\rho^{\prime}},
\end{gathered}
$$

for a choice of the form $N_{1}>k_{1}+n, N_{2}>k_{2}+n, M_{1}>s_{1}\left(N_{1}, N_{2}\right)+n, M_{2}>s_{2}\left(N_{1}, N_{2}\right)+n$.

In order to finish the proof we only have to apply a derivation operator of the form $\partial_{x}^{a} \partial_{\xi}^{\alpha}$ to our function $G(\epsilon ; \lambda ;(x, \xi))$ (defined in (17)) and remark that 
- for any multi-index $b \in \mathbb{N}^{n}$ the function $\left(\partial_{x}^{b} \gamma^{\epsilon}\right)(x ; y, z)$ verifies exactly the same properties as $\gamma^{\epsilon}(x ; y, z)$;

- for any multi-indices $b \in \mathbb{N}^{n}$ and $\beta \in \mathbb{N}^{n}$ the function $\left(\partial_{x}^{b} \partial^{\beta} F\right)_{\lambda}^{\epsilon}(X ; Y, Z)$ verifies exactly the same properties as $F_{\lambda}^{\epsilon}(X ; Y, Z)$.

Let us remark that under Hypothesis 1.2 the 'magnetic phase factor' $\Omega^{\epsilon}$ in the explicit formula of the Moyal product satisfies the hypothesis on .the functions $\gamma$ in the statement of the above Lemma 3.8

Lemma 3.9. Assume Hypothesis 1.2. Then:

(a) for each $\epsilon \in I$ and $x \in \mathcal{X}, \Omega^{\epsilon}(x ; \cdot, \cdot) \in C_{\text {pol }}^{\infty}(\mathcal{X} \times \mathcal{X})$;

(b) for each $N_{1}, N_{2} \in \mathbb{N}$, and $\alpha \in \mathbb{N}^{n}$ there exist $s_{1}\left(N_{1}, N_{2}\right) \geq 0$ and $s_{2}\left(N_{1}, N_{2}\right) \geq 0$ such that

$$
\sup _{\epsilon \in I}\left\|\partial_{x}^{\alpha} \Omega^{\epsilon}\right\|_{\infty,\left(\mathcal{X}, s_{1}\left(N_{1}, N_{2}\right), N_{1}\right),\left(\mathcal{X}, s_{2}\left(N_{1}, N_{2}\right), N_{2}\right)}<\infty
$$

(c) the map $I \ni \epsilon \mapsto \Omega^{\epsilon} \in B C^{\infty}\left(\mathcal{X} ; C_{\mathrm{pol}}^{\infty}(\mathcal{X} \times \mathcal{X})\right)$ is continuous.

Proof. We use the explicit parametric form of $\Omega^{\epsilon}$ in (6). Taking into account Hypothesis 1.2 , a simple examination of (6) leads directly to the results. See also the proof of Lemma $4.2 \mathrm{in}^{12}$.

We come now to the main technical result of our paper.

Proposition 3.10. Under Hypothesis 1.1 and 1.2, there exists some a $>0$ large enough such that for any $\mathfrak{z} \in \mathbb{C} \backslash[a,+\infty)$ we have:

1. for any $\epsilon \in I$, the function $h_{\epsilon}-\mathfrak{z} 1 \in S_{1}^{m}(\Xi) \subset \mathfrak{M}^{\epsilon}(\Xi)$ is invertible for the $\sharp^{\epsilon}$-product having an inverse $r_{\mathfrak{z}}^{\epsilon} \in \mathfrak{F}[\mathfrak{L}]$;

2. moreover the function $I \times \Xi \ni(\epsilon, X) \mapsto \widetilde{r}_{\mathfrak{z}}(\epsilon, X):=r_{\mathfrak{z}}^{\epsilon}(X)$ belongs to the algebra $\mathfrak{F}[\widetilde{\mathcal{L}}]$ and $\mathfrak{e}_{\epsilon}\left(\widetilde{r}_{\mathfrak{z}}\right)=r_{\mathfrak{z}}^{\epsilon}$.

Proof. For $\lambda>0$ set $f^{\epsilon}:=h^{\epsilon}+\lambda$, consider the point-wise inverse $\left(f^{\epsilon}\right)^{-1}=\left(h^{\epsilon}+\lambda\right)^{-1} \in$ $S_{1}^{-m}(\Xi)$ and compute (in the sense of distributions and using oscillatory integral techniques relying on $\exp [-2 i \sigma(Y, Z)])$

$$
\left[f^{\epsilon} \sharp^{\epsilon}\left(f^{\epsilon}\right)^{-1}\right](X)=
$$




$$
\begin{gathered}
=\pi^{-2 n} \int_{\Xi} \int_{\Xi} d Y d Z e^{-2 i \sigma(Y, Z)} \Omega^{\epsilon}(x ; y, z)\left\{1+\left[f^{\epsilon}(X-Y)-f^{\epsilon}(X-Z)\right]\left(f^{\epsilon}\right)^{-1}(X-Z)\right\}= \\
=1+\pi^{-2 n} \int_{\Xi} \int_{\Xi} d Y d Z e^{-2 i \sigma(Y, Z)} \Omega^{\epsilon}(x ; y, z)\left[f^{\epsilon}(X-Y)-f^{\epsilon}(X-Z)\right]\left(f^{\epsilon}\right)^{-1}(X-Z)= \\
=1+\pi^{-2 n} \int_{\Xi} \int_{\Xi} d Y d Z e^{-2 i z \cdot \eta} e^{2 i y \cdot \zeta} \Omega^{\epsilon}(x ; y, z) \times \\
\times \int_{0}^{1} d s\left[(z-y) \cdot\left(\partial_{x} f^{\epsilon}\right)(X-Z+s(Z-Y))+(\zeta-\eta) \cdot\left(\partial_{\xi} f^{\epsilon}\right)(X-Z+s(Z-Y))\right]\left(f^{\epsilon}\right)^{-1}(X-Z) .
\end{gathered}
$$

The fact that $\partial_{\xi} f^{\epsilon} \in S_{1}^{m-1}(\Xi)$ is important in the following arguments. Although $\partial_{x} f^{\epsilon}$ only belongs to $S_{1}^{m}(\Xi)$ one can write

$$
\left(z_{j}-y_{j}\right)\left[e^{-2 i z \cdot \eta} e^{2 i y \cdot \zeta}\right]=-\frac{1}{2 i}\left(\partial_{\eta_{j}}+\partial_{\zeta_{j}}\right)\left[e^{-2 i z \cdot \eta} e^{2 i y \cdot \zeta}\right]
$$

and after integrating by parts we get the same type of improvement. Thus

$$
\left[f^{\epsilon} \sharp^{\epsilon}\left(f^{\epsilon}\right)^{-1}\right](X)=1+\sum_{k=1}^{n} g_{j}(\epsilon ; \lambda ; X),
$$

where

$$
\begin{gathered}
g_{j}(\epsilon ; \lambda ; X):=\pi^{-2 n} \int_{\Xi} \int_{\Xi} d Y d Z e^{-2 i z \cdot \eta} e^{2 i y \cdot \zeta} \Omega^{\epsilon}(x ; y, z) \times \\
\times\left\{\frac{i}{2} \int_{0}^{1} d s\left(\partial_{\xi_{j}} \partial_{x_{j}} f^{\epsilon}\right)(X-Z+s(Z-Y))\left(f^{\epsilon}\right)^{-1}(X-Z)-\right. \\
-i \int_{0}^{1} d s\left(\partial_{x_{j}} f^{\epsilon}\right)(X-Z+s(Z-Y))\left(\partial_{\xi_{j}} f^{\epsilon}\right)(X-Z)\left(f^{\epsilon}\right)^{-2}(X-Z)+ \\
\left.+\int_{0}^{1} d s\left(\zeta_{j}-\eta_{j}\right)\left(\partial_{\xi_{j}} f^{\epsilon}\right)(X-Z+s(Z-Y))\left(f^{\epsilon}\right)^{-1}(X-Z)\right\}= \\
=: \pi^{-2 n} \int_{\Xi} \int_{\Xi} d Y d Z e^{-2 i z \cdot \eta} e^{2 i y \cdot \zeta} \Omega^{\epsilon}(x ; y, z) F_{\lambda}^{\epsilon}(X ; Y, Z) .
\end{gathered}
$$

We intend to prove that each term $g_{j}(\epsilon ; \lambda ; \cdot)$ is a symbol of strictly negative order with a uniform bound controlled by $\lambda>0$. One has

$$
\left[\partial_{x}^{a} \partial_{\xi}^{\alpha} g_{j}\right](\epsilon ; \lambda ; X)=\sum_{b \leq a} C_{a}^{b} \pi^{-2 n} \int_{\Xi} \int_{\Xi} d Y d Z e^{-2 i z \cdot \eta} e^{2 i y \cdot \zeta}\left[\partial_{x}^{a-b} \Omega^{\epsilon}\right](x ; y, z)\left[\partial_{x}^{b} \partial_{\xi}^{\alpha} F_{\lambda}^{\epsilon}\right](X ; Y, Z)
$$

Due to our Hypothesis 1.2, all the derivatives $\partial_{x}^{c} \Omega^{\epsilon}$ are dealt with by Lemma 3.9. We now study the functions $\partial_{x}^{b} \partial_{\xi}^{\alpha} F^{\epsilon}$ with $F^{\epsilon}$ defined in (19); any of these functions is the sum of three contributions. 
- Let us begin with

$$
\begin{gathered}
\partial_{x}^{b} \partial_{\xi}^{\alpha}\left[\left(\partial_{\xi_{j}} \partial_{x_{j}} f^{\epsilon}\right)(X-Z+s(Z-Y))\left(f^{\epsilon}\right)^{-1}(X-Z)\right]= \\
=\sum_{c \leq b, \beta \leq \alpha} C_{\alpha, \beta}^{b, c}\left[\partial_{x}^{b-c} \partial_{\xi}^{\alpha-\beta} \partial_{\xi_{j}} \partial_{x_{j}} f^{\epsilon}\right](X-Z+s(Z-Y))\left[\partial_{x}^{c} \partial_{\xi}^{\beta}\left(f^{\epsilon}\right)^{-1}\right](X-Z) .
\end{gathered}
$$

We have to use the fact that, due to the ellipticity of $h,\left|\left(f^{\epsilon}\right)^{-1}(X)\right| \leq C(f)\left(\langle\xi\rangle^{m}+\lambda\right)^{-1}$. Moreover, it is straightforward to see by induction that for $|c|+|\beta| \geq 1$

$$
\partial_{x}^{c} \partial_{\xi}^{\beta}\left(f^{\epsilon}\right)^{-1}=\left(f^{\epsilon}\right)^{-1} \mathfrak{s}_{c, \beta}^{\epsilon}(f), \quad \text { with } \mathfrak{s}_{c, \beta}(f) \in C\left(I ; S_{1}^{-|\beta|}(\Xi)\right)
$$

verifying

$$
\left\|\mathfrak{s}_{c, \beta}(f)\right\|_{\Xi,-|\beta|, N, M} \leq C\left\|f^{\epsilon}\right\|_{\Xi, m, N+|c|, M+|\beta|}^{|c|+|\beta|} .
$$

For $|b|+|\alpha|=0$ we have

$$
\begin{gathered}
\left|\left[\partial_{\xi_{j}} \partial_{x_{j}} f^{\epsilon}\right](X-Z+s(Z-Y))\left[\left(f^{\epsilon}\right)^{-1}\right](X-Z)\right| \leq \\
\leq C(f)\left\|f^{\epsilon}\right\|_{\Xi, m, 1,1} \frac{\langle\xi\rangle^{m-1}\langle\zeta\rangle^{m-1}\langle\eta\rangle^{m-1}}{\langle\xi-\zeta\rangle^{m}+\lambda} \leq C(f)\left\|f^{\epsilon}\right\|_{\Xi, m, 1,1} \frac{\langle\xi\rangle^{m-1}\langle\zeta\rangle^{m-1}\langle\eta\rangle^{m-1}}{\langle\xi\rangle^{m} /\langle\zeta\rangle^{m}+\lambda} \leq \\
\leq C(f)\left\|f^{\epsilon}\right\|_{\Xi, m, 1,1} \frac{\langle\zeta\rangle^{2 m-1}\langle\eta\rangle^{m-1}\langle\xi\rangle^{m-1}}{\langle\xi\rangle^{m}+\lambda\langle\zeta\rangle^{m}} \leq C(f)\left\|f^{\epsilon}\right\|_{\Xi, m, 1,1} \frac{\langle\zeta\rangle^{2 m-1}\langle\eta\rangle^{m-1}\langle\xi\rangle^{m-1}}{\langle\xi\rangle^{m}+\lambda} .
\end{gathered}
$$

We use now the inequality $a+b \geq(\mu a)^{\mu^{-1}}(\nu b)^{\nu^{-1}}$, valid for $a, b \in \mathbb{R}_{+}, \mu, \nu \in[1,+\infty), \mu^{-1}+$ $\nu^{-1}=1$. Thus for some $\nu \geq 1$ such that $m \nu^{-1}-1<0$, we have

$$
\begin{gathered}
\left|\left[\partial_{\xi_{j}} \partial_{x_{j}} f^{\epsilon}\right](X-Z+s(Z-Y))\left[\left(f^{\epsilon}\right)^{-1}\right](X-Z)\right| \leq \\
\leq C(f)\left\|f^{\epsilon}\right\|_{\Xi, m, 1,1}\langle\zeta\rangle^{2 m-1}\langle\eta\rangle^{m-1} \frac{\langle\xi\rangle^{m-1}}{\langle\xi\rangle^{m}+\lambda} \leq \\
\leq C(f) C(\nu)\left\|f^{\epsilon}\right\|_{\Xi, m, 1,1}\langle\zeta\rangle^{2 m-1}\langle\eta\rangle^{m-1}\langle\xi\rangle^{m \nu^{-1}-1} \lambda^{-\nu^{-1}}
\end{gathered}
$$

For any fixed $X \in \Xi$ we have, by very similar arguments, the following estimations:

$$
\begin{gathered}
\left|\partial_{y}^{b} \partial_{\eta}^{\alpha}\left[\left(\partial_{\xi_{j}} \partial_{x_{j}} f^{\epsilon}\right)(X-Z+s(Z-Y))\left[\left(f^{\epsilon}\right)^{-1}\right](X-Z)\right]\right| \leq \\
\leq C(f) C(\nu)\left\|f^{\epsilon}\right\|_{\Xi, m, 1,1}\langle\zeta\rangle^{2 m-1-|\alpha|}\langle\eta\rangle^{m-1-|\alpha|}\langle\xi\rangle^{m \nu^{-1}-1-|\alpha|} \lambda^{-\nu^{-1}} .
\end{gathered}
$$

Let us remark that

$$
\partial_{z}^{b} \partial_{\zeta}^{\alpha}\left[\left(\partial_{\xi_{j}} \partial_{x_{j}} f^{\epsilon}\right)(X-Z+s(Z-Y))\left[\left(f^{\epsilon}\right)^{-1}\right](X-Z)\right]=
$$


$=(-1)^{|\alpha|} \sum_{c \leq b, \beta \leq \alpha} C_{\alpha, \beta}^{b, c}(1-s)^{|\alpha|-|\beta|}\left[\partial_{x}^{b-c} \partial_{\xi}^{\alpha-\beta} \partial_{\xi_{j}} \partial_{x_{j}} f^{\epsilon}\right](X-Z+s(Z-Y))\left[\partial_{x}^{c} \partial_{\xi}^{\beta}\left(f^{\epsilon}\right)^{-1}\right](X-Z)$,

and thus is completely similar to the higher order derivatives $\partial_{x}^{b} \partial_{\xi}^{\alpha}$ that we shall now study.

For $|b|+|\alpha|>0$ we obtain

$$
\begin{gathered}
\left|\partial_{x}^{b} \partial_{\xi}^{\alpha}\left[\left(\partial_{\xi_{j}} \partial_{x_{j}} f^{\epsilon}\right)(X-Z+s(Z-Y))\left(f^{\epsilon}\right)^{-1}(X-Z)\right]\right| \leq \\
\leq\left|f_{\epsilon}^{-1}(X-Z)\right| \sum_{c \leq b} C_{b}^{c} \sum_{\beta \leq \alpha} C_{\alpha}^{\beta}\left\|f^{\epsilon}\right\|_{\Xi, m,|b-c|+1,|\alpha-\beta|+1}[\langle\xi\rangle\langle\eta\rangle\langle\zeta\rangle]^{m-|\alpha-\beta|-1}\left|\mathfrak{s}_{c, \beta}(f)(X-Z)\right| \leq \\
\leq C(b, \alpha)\left\|f^{\epsilon}\right\|_{\Xi, m,|b|+1,|\alpha|+1}^{|b|+1+|\alpha|+1} \frac{[\langle\xi\rangle\langle\eta\rangle\langle\zeta\rangle]^{m-|\alpha|-1}}{\langle\xi-\zeta\rangle^{m}+\lambda} \leq \\
\leq C(b, \alpha) C(\nu)\left\|f^{\epsilon}\right\|_{\Xi, m,|b|+1,|\alpha|+1}^{|b|+1+|\alpha|+1}\langle\eta\rangle^{m-|\alpha|-1}\langle\zeta\rangle^{2 m-|\alpha|-1}\langle\xi\rangle^{m \nu^{-1}-1-|\alpha|} \lambda^{-\nu^{-1}}
\end{gathered}
$$

and similar estimations for this term after application of differential operators of the form $\partial_{y}^{b} \partial_{\eta}^{\alpha}$ or $\partial_{z}^{b} \partial_{\zeta}^{\alpha}$. Thus, for each $\epsilon \in I$ this first term (that we denote by $F_{\lambda, 1}^{\epsilon}$ ) satisfies:

$$
F_{\lambda, 1}^{\epsilon} \in B C^{\infty}\left(\Xi ; S_{1,1}^{m-1,2 m-1}(\Xi \times \Xi)\right)
$$

and

$$
\begin{gathered}
\operatorname{supsup}_{\epsilon \in I}\left\|\left[\partial_{x}^{b} \partial_{\xi}^{\alpha} F_{\lambda, 1}^{\epsilon}\right](X ; \cdot, \cdot)\right\|_{\left(m-1, N_{1}, M_{1}\right),\left(2 m-1, N_{2}, M_{2}\right)} \leq \\
\leq C(b, \alpha) C(\nu) C(f)\left\|f^{\epsilon}\right\|_{\Xi, m,|b|+N_{1}+N_{2}+1,|\alpha|+M_{1}+M_{2}+1}^{2+|b|+|\alpha|+N_{2}+M_{2}}\langle\xi\rangle^{m \nu^{-1}-1-|\alpha|} \lambda^{-\nu^{-1}}
\end{gathered}
$$

Let us study its continuity with respect to $\epsilon \in I$. First, we fix some $\epsilon \in I$ and for any $\epsilon^{\prime} \in I$ we consider the difference

$$
\begin{gathered}
\left(\partial_{\xi_{j}} \partial_{x_{j}} f^{\epsilon^{\prime}}\right)(X-Z+s(Z-Y))\left(f^{\epsilon^{\prime}}\right)^{-1}(X-Z)-\left(\partial_{\xi_{j}} \partial_{x_{j}} f^{\epsilon}\right)(X-Z+s(Z-Y)) f_{\epsilon}^{-1}(X-Z)= \\
=\left[\left(\partial_{\xi_{j}} \partial_{x_{j}}\right)\left(f^{\epsilon^{\prime}}-f_{\epsilon}\right)(X-Z+s(Z-Y))\right]\left(f^{\epsilon^{\prime}}\right)^{-1}(X-Z)+ \\
+\left(\partial_{\xi_{j}} \partial_{x_{j}} f^{\epsilon}\right)(X-Z+s(Z-Y))\left[\left(f^{\epsilon^{\prime}}\right)^{-1}-\left(f^{\epsilon}\right)^{-1}\right](X-Z)= \\
=\left[\left(\partial_{\xi_{j}} \partial_{x_{j}}\right)\left(f^{\epsilon^{\prime}}-f^{\epsilon}\right)(X-Z+s(Z-Y))\right]\left(f^{\epsilon^{\prime}}\right)^{-1}(X-Z)+ \\
+\left(\partial_{\xi_{j}} \partial_{x_{j}} f^{\epsilon}\right)(X-Z+s(Z-Y))\left[\left(f^{\epsilon^{\prime}}\right)^{-1}\left(f^{\epsilon^{\prime}}-f^{\epsilon}\right)\left[\left(f^{\epsilon}\right)^{-1}\right]\right](X-Z) .
\end{gathered}
$$

After applying the operator $\partial_{x}^{a} \partial_{\xi}^{\alpha}$ to the above difference, for the first term we can directly use the previous analysis with $f^{\epsilon}$ replaced by $f^{\epsilon^{\prime}}-f^{\epsilon}$ and obtain a uniform bound with some constant multiplied by

$$
\left\|f^{\epsilon^{\prime}}-f^{\epsilon}\right\|_{\Xi, m,|b|+1,|\alpha|+1}^{|b|+1+|\alpha|+1}
$$


that converges to zero for $\epsilon^{\prime} \rightarrow \epsilon$ due to our Hypothesis 1.1. For the second term, we have to replace in the previous analysis the factor $\left(\partial_{\xi_{j}} \partial_{x_{j}} f^{\epsilon}\right)(X-Z+s(Z-Y))$ that is an element of $S_{1}^{m-1}(\Xi)$ and thus satisfies an estimation of the type

$$
\left(\partial_{x}^{b} \partial_{\xi}^{\alpha} \partial_{\xi_{j}} \partial_{x_{j}} f^{\epsilon}\right)(X-Z+s(Z-Y)) \leq\left\|f^{\epsilon}\right\|_{\Xi, m,|b|+1,|\alpha|+1}\langle\xi\rangle^{m-1-|\alpha|}\langle\eta\rangle^{m-1-|\alpha|}\langle\zeta\rangle^{m-1-|\alpha|}
$$

with the derivatives $\partial_{x}^{b} \partial_{\xi}^{\alpha}$ of the factor

$$
\left(\partial_{\xi_{j}} \partial_{x_{j}} f^{\epsilon}\right)(X-Z+s(Z-Y))\left(f^{\epsilon^{\prime}}\right)^{-1}(X-Z)\left(f^{\epsilon^{\prime}}-f^{\epsilon}\right)(X-Z)
$$

that are bounded by

$$
C(b, \alpha)\left\|f^{\epsilon^{\prime}}-f^{\epsilon}\right\|_{\Xi, m,|b|+1,|\alpha|+1}\left\|f^{\epsilon}\right\|_{\Xi, m,|b|+1,|\alpha|+1}^{3+|b|+|\alpha|}\langle\xi\rangle^{m-1-|\alpha|}\langle\eta\rangle^{m-1-|\alpha|}\langle\zeta\rangle^{3 m-1-|\alpha|} .
$$

Similar estimations are obtained analogously, applying differential operators of the form $\partial_{y}^{b} \partial_{\eta}^{\alpha}$ or $\partial_{z}^{b} \partial_{\zeta}^{\alpha}$. Thus

$$
\begin{gathered}
\sup _{x \in \mathcal{X}}\left\|\left[\partial_{x}^{b} \partial_{\xi}^{\alpha}\left(F_{\lambda, 1}^{\epsilon}-F_{\lambda, 1}^{\epsilon}\right)\right](X ; \cdot, \cdot)\right\|_{\left(m-1, N_{1}, M_{1}\right),\left(3 m-1, N_{2}, M_{2}\right)} \leq \\
\leq C(b, \alpha)\left\|f^{\epsilon}\right\|_{\Xi, m,|b|+N_{1}+N_{2}+1,|\alpha|+M_{1}+M_{2}+1}^{3+|b|+|\alpha|+N_{2}+M_{2}}\left\|f^{\epsilon^{\prime}-f^{\epsilon}}\right\|_{\Xi, m,|b|+N_{1}+N_{2}+1,|\alpha|+M_{1}+M_{2}+1} .
\end{gathered}
$$

We conclude that the first term in (19) satisfies the hypothesis for $F$ in Lemma 3.8.

- Now let us consider the second term in (19):

$$
\begin{gathered}
\left(\partial_{x_{j}} f^{\epsilon}\right)(X-Z+s(Z-Y))\left(\partial_{\xi_{j}} f^{\epsilon}\right)(X-Z) f_{\epsilon}^{-2}(X-Z)= \\
=\left(\partial_{x_{j}} f^{\epsilon}\right)(X-Z+s(Z-Y))\left[\left(\partial_{\xi_{j}} f^{\epsilon}\right)(X-Z) f_{\epsilon}^{-1}(X-Z)\right] f_{\epsilon}^{-1}(X-Z) .
\end{gathered}
$$

It verifies the estimation

$$
\begin{gathered}
\left|\left(\partial_{x_{j}} f^{\epsilon}\right)(X-Z+s(Z-Y))\left(\partial_{\xi_{j}}\left(f^{\epsilon}\right)^{-1}\right)(X-Z)\right| \leq \\
\leq C(f)\left|\left(f^{\epsilon}\right)^{-1}(X-Z)\right|\left\|f^{\epsilon}\right\|_{\Xi, m, 1,0}\left\|f^{\epsilon}\right\|_{\Xi, m, 0,1} \frac{\langle\xi\rangle^{m}\langle\eta\rangle^{m}\langle\zeta\rangle^{m}}{\langle\xi\rangle /\langle\zeta\rangle} \leq \\
\leq C(f)\left\|f^{\epsilon}\right\|_{\Xi, m, 1,0}\left\|f^{\epsilon}\right\|_{\Xi, m, 0,1}\langle\eta\rangle^{m}\langle\zeta\rangle^{2 m+1} \frac{\langle\xi\rangle^{m-1}}{\langle\xi\rangle^{m}+\lambda} \leq \\
\leq C(\nu) C(f)\left\|f^{\epsilon}\right\|_{\Xi, m, 1,0}\left\|f^{\epsilon}\right\|_{\Xi, m, 0,1}\langle\eta\rangle^{m}\langle\zeta\rangle^{2 m+1}\langle\xi\rangle^{m \nu^{-1}-1} \lambda^{-\nu^{-1}} .
\end{gathered}
$$

Applying then the derivation operator $\partial_{x}^{a} \partial_{\xi}^{\alpha}$ we proceed exactly as for the first term. In fact the essential step is the difference of one unit between the denominator and the numerator in $\langle\xi\rangle^{m-1}\left(\langle\xi\rangle^{m}+\lambda\right)^{-1}$ that was obtained for the first term due to the factor $\partial_{x_{j}} \partial_{\xi_{j}} f^{\epsilon}$ and for this second term from the factor $\left[\left(\partial_{\xi_{j}} f^{\epsilon}\right)(X-Z) f_{\epsilon}^{-1}(X-Z)\right]$ (a strictly positive difference would have been enough). The $\epsilon$-continuity also follows by the same procedure. 
- Now let us consider the third term in (19):

$$
\left(\zeta_{j}-\eta_{j}\right)\left(\partial_{\xi_{j}} f^{\epsilon}\right)(X-Z+s(Z-Y))\left(f^{\epsilon}\right)^{-1}(X-Z) .
$$

Recalling the above observation we notice that the same type of factor $\langle\xi\rangle^{m-1}\left(\langle\xi\rangle^{m}+\lambda\right)^{-1}$ will now be obtained due to the presence of the factor $\partial_{\xi_{j}} f^{\epsilon}$. The presence of the factor $\left(\zeta_{j}-\eta_{j}\right)$ will only contribute to modify the order of symbols in the given variables so that we shall obtain the estimation

$$
\begin{gathered}
\left|\left(\zeta_{j}-\eta_{j}\right)\left(\partial_{\xi_{j}} f^{\epsilon}\right)(X-Z+s(Z-Y))\left(f^{\epsilon}\right)^{-1}(X-Z)\right| \leq \\
\leq C(\nu) C(f)\left\|f^{\epsilon}\right\|_{\Xi, m, 0,1}\langle\eta\rangle^{m}\langle\zeta\rangle^{2 m}\langle\xi\rangle^{m \nu^{-1}-1} \lambda^{-\nu^{-1}} .
\end{gathered}
$$

Obviously all the following arguments given for the first term still remain true for this third term in (19).

We conclude that

$$
\begin{gathered}
\sup _{\epsilon \in I} \sup _{x \in \mathcal{X}}\left\|\left[\partial_{x}^{b} \partial_{\xi}^{\alpha} F_{\lambda}^{\epsilon}\right](X ; \cdot, \cdot)\right\|_{\left(m, N_{1}, M_{1}\right),\left(2 m+1, N_{2}, M_{2}\right)} \leq \\
\leq C(\nu) C(f)\left\|f^{\epsilon}\right\|_{\Xi, m,|b|+N_{1}+N_{2}+1,|\alpha|+M_{1}+M_{2}+1}^{2+|b|+|\alpha|+N_{2}+M_{2}}\langle\xi\rangle^{m \nu^{-1}-1} \lambda^{-\nu^{-1}} .
\end{gathered}
$$

Thus, choosing $\nu>m$, we have $\rho:=-m \nu^{-1}+1>0$ and thus we can use Lemma 3.8 to deduce that for each $\lambda>0$ the application $\boldsymbol{g}(\epsilon ; \lambda ; X):=\sum_{1 \leq j \leq n} g_{j}(\epsilon ; \lambda ; X)$ defines an element $\boldsymbol{g}(\cdot ; \lambda ; \cdot) \in C\left(I ; S_{1}^{-\rho}(\Xi)\right)$ and moreover we have for any $\nu>m$

$$
\sup _{\epsilon \in I}\|\boldsymbol{g}(\epsilon ; \lambda ; \cdot)\|_{\Xi, 1,-\rho, N, M} \leq C_{\nu} \lambda^{-\nu} .
$$

Thus, we conclude that for any $\alpha \in \mathbb{N}^{n}$ there exists a constant $C_{\alpha, \nu}$ such that

$$
\operatorname{supsupsup}_{\epsilon \in I}\left|<\xi \mathcal{X}_{\xi \in \mathcal{X}^{\prime}}>^{(\rho-|\alpha|)}\left(\partial_{\xi}^{\alpha} \boldsymbol{g}\right)(\epsilon ; \lambda ; x, \xi)\right| \leq C_{\alpha, \nu} \lambda^{-\nu} .
$$

Fixing $\epsilon \in I$ and using Lemma A.4 in $^{18}$ and the argument at the end of the proof of Theorem 1.8 ( ${ }^{18}$ Section 2.1), one obtains the conclusion of point 1 of the Proposition.

A completely straightforward modification of Lemma A.4 in ${ }^{18}$ (just take $(\epsilon, x) \in I \times \mathcal{X}$ instead of $q \in \mathcal{X}$ in the proof given in $^{18}$ ) allows us to conclude that for any $\lambda>0$ and any $\epsilon \in I$ we have that (for any $\nu>m$ )

$$
\left(\mathfrak{F}^{-1} \boldsymbol{g}\right)(\cdot ; \lambda ; \cdot) \in L^{1}\left(\left(\mathcal{X} ; C\left(I ; B C_{u}(\mathcal{X})\right)\right)=\widetilde{\mathcal{L}} \quad \text { and } \quad\left\|\left(\mathfrak{F}^{-1} \boldsymbol{g}\right)(\cdot ; \lambda ; \cdot)\right\|_{\widetilde{\mathcal{L}}} \leq C_{\nu} \lambda^{-\nu}\right.
$$


Using (18) and the above result and repeating the arguments at the end of the proof of Theorem 1.8 ( ${ }^{18}$ Section 2.1), we obtain also the second point of the proposition.

Corollary 3.11. The map

$$
I \ni \epsilon \mapsto r_{\mathfrak{z}}^{\epsilon} \in \mathfrak{F}[\mathfrak{L}]
$$

is continuous for the topology induced by the norm $\|G\|_{\mathfrak{F}(\mathfrak{L})}:=\int_{\mathcal{X}} d x\|[\mathfrak{F}(G)](x)\|_{\infty}$.

Proof. We notice that for any $\widetilde{\phi} \in C_{c}\left(\mathcal{X} ; C\left(I ; B C_{u}(\mathcal{X})\right)\right)$ we have

$$
\sup _{\epsilon \in I} \int_{\mathcal{X}} d x\|[\widetilde{\phi}(x)](\epsilon)\|_{B C_{u}(\mathcal{X})} \leq \int_{\mathcal{X}} d x \sup _{\epsilon \in I}\|[\widetilde{\phi}(x)](\epsilon)\|_{B C_{u}(\mathcal{X})}
$$

and thus $\widetilde{\mathcal{L}} \subset C(I ; \mathcal{L})$.

\section{A CONTINUOUS FIELD OF TWISTED CROSSED PRODUCTS}

In this section we prove Theorem 2.7, which in its turn implies our main result. Our proposal is to use the concept of continuous field of $C^{*}$-algebras ${ }^{5,7,9,20,22-25}$, and Theorem 2.4 of ${ }^{23}$. Let us notice that we are in the frame of Section $2 \mathrm{of}^{23}$ with the $C^{*}$-algebra $A=B C_{u}(\mathcal{X})$ (that is abelian and unital), the locally compact space $\Omega=I$ (that is even compact in our case) and the locally compact group $G=\mathcal{X} \cong \mathbb{R}^{n}$ that is abelian and second countable. The action of the group on the $C^{*}$-algebra is explicitely given as the action $\theta$ of $\mathcal{X}$ on $B C_{u}(\mathcal{X})$ by translations. Let us recall the notion of continuous field of $\theta$-cocycles on the group $\mathcal{X}$ over the locally compact space $I$, as introduced in ${ }^{23}$ :

Definition 4.12. A continuous field of $\theta$-cocycles on $\mathcal{X}$ over $I$ is a function

$$
\widetilde{\omega}: I \times \mathcal{X} \times \mathcal{X} \rightarrow B C_{u}(\mathcal{X})
$$

such that:

1. for any $\epsilon \in I$ the map $\widetilde{\omega}(\epsilon, \cdot, \cdot)$ defines a normalized $\theta$-cocycle on $\mathcal{X}$, i.e. $|[\widetilde{\omega}(\epsilon, y, z)](x)|=1$ and

$$
\widetilde{\omega}(\epsilon, x, y+z) \theta_{x}[\widetilde{\omega}(\epsilon, y, z)]=\widetilde{\omega}(\epsilon, x+y, z) \widetilde{\omega}(\epsilon, x, y), \quad \widetilde{\omega}(\epsilon, x, 0)=\widetilde{\omega}(\epsilon, 0, x)=1 ;
$$

2. for any $(y, z) \in \mathcal{X} \times \mathcal{X}$ the map $I \ni \epsilon \mapsto \widetilde{\omega}(\epsilon, y, z) \in B C_{u}(\mathcal{X})$ is continuous;

3. the map $\mathcal{X} \times \mathcal{X} \ni(y, z) \mapsto \widetilde{\omega}(\cdot, y, z) \in C\left(I ; B C_{u}(\mathcal{X})\right)$ is Bochner measurable. 
Remark 4.13. The map $\widetilde{\omega}$ defined in (13) and (11) satisfies the conditions for a continuous field of $\theta$-cocycles on $\mathcal{X}$ over $I$. One checks easily that the first condition is satisfied, by an inspection of the explicit definition; the last two conditions follow from Hypothesis 1.2 that implies that $\tilde{\omega}$ belongs in fact to $C\left(\mathcal{X} \times \mathcal{X} ; C\left(I ; B C_{u}(\mathcal{X})\right)\right)$.

In this framework we follow M. Rieffel ${ }^{23}$ and consider the field of $C^{*}$-algebras $\left\{\mathfrak{B}^{\epsilon}\right\}_{\epsilon \in I}$ over the compact space $I$ and $\mathfrak{B}$ as a $C^{*}$-algebra of cross-sections of this field. Then combining our framework with Theorem 2.4 and Propositions 1.2 and $2.3 \mathrm{in}^{23}$, one obtains the following result:

Proposition 4.14. The family of maps $\left\{\mathfrak{e}_{\epsilon}: \mathfrak{B} \rightarrow \mathfrak{B}^{\epsilon}\right\}_{\epsilon \in I}$ have the following properties:

1. each $\mathfrak{e}_{\epsilon}: \mathfrak{B} \rightarrow \mathfrak{B}^{\epsilon}$ is surjective;

2. for any $F \in \mathfrak{B}$ we have $\|F\|_{\mathfrak{B}}=\sup _{\epsilon \in I}\left\|\mathfrak{e}_{\epsilon}(F)\right\|_{\mathfrak{B}^{\epsilon}}$;

3. for any $F \in \mathfrak{B}$ the map $I \ni \epsilon \mapsto\left\|\mathfrak{e}_{\epsilon}(F)\right\|_{\mathfrak{B}^{\epsilon}} \in \mathbb{R}_{+}$is upper semi-continuous.

Our Proposition 3.10 now evidently implies the following Corollary

Corollary 4.15. Under our Hypothesis 1.1 and 1.2, the map $I \ni \epsilon \mapsto\left\|r_{\mathfrak{z}}^{\epsilon}\right\|_{\mathfrak{B}^{\epsilon}}$ is upper semi-continuous.

Using now our Corollary 3.11 and the Hilbert space representation (12) we shall obtain the lower semi-continuity of our map $I \ni \epsilon \mapsto\left\|r_{\mathfrak{z}}^{\epsilon}\right\|_{\epsilon}$ by a standard procedure.

Lemma 4.16. Given a continuous function $I \ni \epsilon \mapsto \phi^{\epsilon} \in \mathfrak{L}$ and an element $\psi \in \mathcal{H}$, the map

$$
I \ni \epsilon \mapsto \phi^{\epsilon} \diamond^{\epsilon} \psi \in \mathcal{H}
$$

is continuous.

Proof. For $\epsilon, \epsilon^{\prime} \in I$ we have

$$
\left\|\phi^{\epsilon} \diamond^{\epsilon} \psi-\phi^{\epsilon^{\prime}} \diamond^{\epsilon^{\prime}} \psi\right\|_{L^{2}(\mathcal{X} \times \mathcal{X})} \leq\left\|\phi^{\epsilon} \diamond^{\epsilon} \psi-\phi^{\epsilon^{\prime} \diamond^{\epsilon}} \psi\right\|_{L^{2}(\mathcal{X} \times \mathcal{X})}+\left\|\phi^{\epsilon^{\prime}} \diamond^{\epsilon} \psi-\phi^{\epsilon^{\prime} \diamond^{\epsilon^{\prime}}} \psi\right\|_{L^{2}(\mathcal{X} \times \mathcal{X})} .
$$

To estimate the first term we use the definition of the $\diamond^{\epsilon}$ product in order to write

$$
\left[\phi^{\epsilon} \diamond^{\epsilon} \psi-\phi^{\epsilon^{\prime}} \diamond^{\epsilon} \psi\right](q, x)=\int_{\mathcal{X}} d y\left(\phi^{\epsilon}-\phi^{\epsilon^{\prime}}\right)\left(q-\frac{x-y}{2} ; y\right) \psi\left(q+\frac{y}{2} ; x-y\right) \omega^{\epsilon}\left(q-\frac{x}{2} ; y, x-y\right)
$$

So

$\left\|\phi^{\epsilon} \diamond^{\epsilon} \psi-\phi^{\epsilon^{\prime}} \diamond^{\epsilon} \psi\right\|_{L^{2}(\mathcal{X} \times \mathcal{X})}^{2} \leq \int_{\mathcal{X}} d q \int_{\mathcal{X}} d x\left[\int_{\mathcal{X}} d y\left|\left(\phi^{\epsilon}-\phi^{\epsilon^{\prime}}\right)\left(q-\frac{x-y}{2} ; y\right) \psi\left(q+\frac{y}{2} ; x-y\right)\right|\right]^{2} \leq$ 


$$
\begin{gathered}
\leq \int_{\mathcal{X}} d q \int_{\mathcal{X}} d x\left[\int_{\mathcal{X}} d y\left(\sup _{z \in \mathcal{X}}\left|\left(\phi^{\epsilon}-\phi^{\epsilon^{\prime}}\right)(z ; y)\right|\right)\left|\psi\left(q+\frac{y}{2} ; x-y\right)\right|\right]^{2} \leq \\
\leq\left\|\phi^{\epsilon}-\phi^{\epsilon^{\prime}}\right\|_{1, \infty} \int_{\mathcal{X}} d q \int_{\mathcal{X}} d x \int_{\mathcal{X}} d y\left(\sup _{z \in \mathcal{X}}\left|\left(\phi^{\epsilon}-\phi^{\epsilon^{\prime}}\right)(z ; y)\right|\right)\left|\psi\left(q+\frac{y}{2} ; x-y\right)\right|^{2}= \\
=\left\|\phi^{\epsilon}-\phi^{\epsilon^{\prime}}\right\|_{\mathfrak{L}}^{2}\|\psi\|_{L^{2}(\mathcal{X} \times \mathcal{X})}^{2} \underset{\epsilon^{\prime} \rightarrow \epsilon}{\longrightarrow} 0
\end{gathered}
$$

using Fubini and a change of variables. It remains to verify that second term in (23) also converges to 0 ; one has:

$$
\begin{gathered}
\left\|\phi^{\epsilon^{\prime}} \diamond^{\epsilon} \psi-\phi^{\epsilon^{\prime}} \diamond^{\epsilon^{\prime}} \psi\right\|_{L^{2}(\mathcal{X} \times \mathcal{X})}^{2} \leq \\
\leq \int_{\mathcal{X}} d q \int_{\mathcal{X}} d x\left[\int_{\mathcal{X}} d y\left|\phi^{\epsilon^{\prime}}\left(q-\frac{x-y}{2} ; y\right) \psi\left(q+\frac{y}{2} ; x-y\right)\right| \times\right. \\
\times\left|\omega^{\epsilon}\left(q-\frac{x}{2} ; y, x-y\right)-\omega^{\epsilon^{\prime}}\left(q-\frac{x}{2} ; y, x-y\right)\right|^{2} \leq \\
\leq 4 \int_{\mathcal{X}} d q \int_{X} d x\left[\int_{\mathcal{X}} d y\left\|\phi^{\epsilon^{\prime}}(\cdot ; y)\right\|_{\infty} \mid \psi\left(q+\frac{y}{2} ; x-y\right)\right]^{2} \leq \\
\leq 4\left\|\phi^{\epsilon^{\prime}}\right\|_{1, \infty} \int_{\mathcal{X}} d q \int_{\mathcal{X}} d x \int_{\mathcal{X}} d y\left\|\phi^{\epsilon^{\prime}}(\cdot ; y)\right\|_{\infty}\left|\psi\left(q+\frac{y}{2} ; x-y\right)\right|^{2}=4\left\|\phi^{\epsilon^{\prime}}\right\|_{\mathfrak{L}}^{2}\|\psi\|_{L^{2}(\mathcal{X} \times \mathcal{X})}^{2},
\end{gathered}
$$

by the same procedure as above. Moreover, due to our Hypothesis 1.2 the difference

$$
\omega^{\epsilon}\left(q-\frac{x}{2} ; y, x-y\right)-\omega^{\epsilon}\left(q-\frac{x}{2} ; y, x-y\right)
$$

converges point-wise to 0 for $\left|\epsilon-\epsilon^{\prime}\right| \rightarrow 0$. We conclude by the Dominated Convergence Theorem.

Corollary 4.17. Under our Hypothesis 1.1 and 1.2, the map $I \ni \epsilon \mapsto\left\|r_{\mathfrak{z}}^{\epsilon}\right\|_{\mathfrak{B}^{\epsilon}}$ is lower semi-continuous.

Proof. We use Corollary 3.11, the above Lemma 4.16 and the well known fact that if a family $\left\{S^{\epsilon}\right\}_{\epsilon \in I}$ of bounded linear operators in a Hilbert space $\mathcal{H}$ is strongly continuous, then $\epsilon \mapsto\left\|S^{\epsilon}\right\|_{\mathbb{B}(\mathcal{H})}$ is lower semi-continuous (as the supremum of a family of continuous functions).

Combining the Corollaries 4.15 and 4.17 one proves Theorem 2.7 and finishes the proof of Theorem 1.4.

Acknowledgements: M. Măntoiu is partially supported by Núcleo Cientifico ICM P0\%027-F "Mathematical Theory of Quantum and Classical Magnetic Systems" and by Chilean Science Foundation Fondecyt under the Grant 1085162. R. Purice has been supported by CNCSIS under the Ideas Programme, PCCE project no. 55/2008 Sisteme diferentiale in 
analiza neliniara si aplicatii. N. Athmouni thanks IMAR Bucharest for its hospitality and Mondher Damak for his continuous support. R. Purice thanks the Universities of Chile and Sfax for their hospitality.

\section{REFERENCES}

${ }^{1}$ W.O. Amrein, A. Boutet de Monvel and V. Georgescu: $\mathcal{C}_{0}$-Groups, Commutator Methods and Spectral Theory of N-Body Hamiltonians, Birkhäuser, Basel, (1996).

${ }^{2}$ J. Avron and B. Simon: Stability of Gaps for Periodic Potentials Under a Variation of the Magnetic Field, J. Phys. A: Math. Gen. 18, 2199-2205, (1985).

${ }^{3}$ J. Bellissard: Lipschitz Continuity of Gap Boundaries for Hofstadter-like Spectra, Commun. Math. Phys. 160, 599-613, (1994).

${ }^{4}$ J. Bellissard, B. Iochum and D. Testard: Continuity Properties of the Electronic Spectrum of $1 D$ Quasicristals, Commun. Math. Phys. 141, 353-380, (1991).

${ }^{5}$ E. Blanchard: Déformation de $C^{*}$-algèbres de Hopf, Bull. Soc. Math. France 124, 141-215, (1996).

${ }^{6} \mathrm{Ph}$. Briet and H.D. Cornean: Locating the Spectrum for Magnetid Schródinger and Dirac Operators, Commun. in P. D. E., 27, no. 5-6, 1079-1101, (2002).

7. J. Dixmier: Les $C^{*}$-algèbres et leurs représentations, Gauthier-Villars, Paris, (1964).

${ }^{8}$ G. Elliott: Gaps in the Spectrum of an Almost Periodic Schrödinger Operator, C. R. Math. Rep. Acad. Sci. Canada, 4, 255-259, (1982).

${ }^{9}$ J. M. G. Fell: The structure of algebras of operator fields, Acta Math., 106, (1961), 233-280.

${ }^{10} \mathrm{~V}$. Georgescu and A. Iftimovici, Crossed Products of $C^{*}$-Algebras and Spectral Analysis of Quantum Hamiltonians, Commun. Math. Phys. 228, 519-560, (2002).

${ }^{11} \mathrm{~V}$. Iftimie: Opérateurs differentiels magnétiques: Stabilité des trous dans le spectre, invariance du spectre essentiel et applications, Commun. in P.D.E. 18, 651-686, (1993).

${ }^{12}$ V. Iftimie, M. Măntoiu and R. Purice: Magnetic Pseudodifferential Operators, Publ. RIMS, 43 No.3, 585-624, (2007).

${ }^{13}$ M.V. Karasev and T.A. Osborn: Symplectic Areas, Quantization and Dynamics in Electromagnetic Fields, J. Math. Phys. 43, 756-788, (2002).

${ }^{14}$ M. Lein, M. Măntoiu and S. Richard: Magnetic Pseudodifferential Operators with Coeffi- 
cients in $C^{*}$-algebras, to appear in Publications of the RIMS.

${ }^{15}$ M. Măntoiu and R. Purice: The Magnetic Weyl Calculus, J. Math. Phys. 45, No.4, 1394$1417,(2004)$.

${ }^{16}$ M. Măntoiu and R. Purice, The Mathematical Formalism of a Particle in a Magnetic Field, in Mathematical Physics of Quantum Mechanics, pp. 417-434, Lecture Notes in Phys. 690, Springer, Berlin, 2006.

${ }^{17}$ M. Măntoiu, R. Purice and S. Richard: Twisted Crossed Products and Magnetic Pseudodifferential Operators, Proceedings of the Conference of Sinaia (Romania), Theta Foundation (2005).

${ }^{18}$ M. Măntoiu, R. Purice and S. Richard: Spectral and Propagation Results for Magnetic Schrödinger Operators; a $C^{*}$-Algebraic Approach, J. Funct. Anal. 250 No.1, 42-67, (2007).

${ }^{19}$ G. Nenciu: Stability of Energy Gaps Under Variations of the Magnetic Field, Letters in Mat. Phys. 11, 127-132, (1986).

${ }^{20}$ M. Nilsen: $C^{*}$-Bundles and $C_{0}(X)$-Algebras, Indiana Univ. Math. J. 45, 463-476, (1996).

${ }^{21}$ J. Packer and I. Raeburn: Twisted Crossed Products of $C^{*}$-Algebras, Math. Proc. Camb. Phil. Soc. 106, 293-311, (1989).

${ }^{22}$ J. Packer and I. Raeburn: Twisted Crossed Products of $C^{*}$-Algebras II, Math. Ann. 287, 595-612, (1990).

${ }^{23}$ M. A. Rieffel, Continuous Fields of $C^{*}$-algebras Coming from Group Cocycles and Actions, Math. Ann. 283, 631-643, (1989).

${ }^{24}$ J. Tomiyama, Topological representation of C-algebras, Tohoku Math. J., (2) 14, (1962), $187-204$.

${ }^{25} \mathrm{~J}$. Tomiyama, A characterization of C-algebras whose conjugate spaces are separable, Tohoku Math. J., (2) 15, (1963), 96-102. 